\title{
Ethik als notwendige Basiskompetenz von Psychotherapeuten
}

Trachsel, Manuel

\begin{abstract}
Da ethische Fragen und Konflikte in der Psychotherapie omnipräsent sind, ist eine Psychotherapie erst dann gut, wenn diese ethischen Aspekte einbezogen werden. Psychotherapie-Ethik als Wissenschaft vom moralischen Urteilen und Handeln in der Psychotherapie ist daher eine wichtige Voraussetzung für professionelles klinisches Arbeiten und sollte als Basiskompetenz von Psychotherapeuten systematisch vermittelt werden.
\end{abstract}

DOI: https://doi.org/10.1055/a-0771-7857

Posted at the Zurich Open Repository and Archive, University of Zurich

ZORA URL: https://doi.org/10.5167/uzh-179660

Journal Article

Accepted Version

Originally published at:

Trachsel, Manuel (2019). Ethik als notwendige Basiskompetenz von Psychotherapeuten. Psychotherapie im Dialog, 20(04):45-48.

DOI: https://doi.org/10.1055/a-0771-7857 
Psychotherapie im Dialog

\section{Ethik als notwendige Basiskompetenz von Psychotherapeuten}

\section{Manuel Trachsel}

PD Dr. phil. Dr. med. Manuel Trachsel

Institut für Biomedizinische Ethik und Medizingeschichte

Medizinische Fakultät

Universität Zürich

Winterthurerstrasse 30

CH-8006 Zürich

Schweiz

Tel. +41786854454

Mailmanuel.trachsel@uzh.ch

Web https://www.ibme.uzh.ch/en/Biomedical-Ethics/Team/Members/manueltrachsel.html 


\section{(Vorspann)}

Wirksamkeit, Zweckmässigkeit und Wirtschaftlichkeit sind notwendige, jedoch nicht hinreichende Kriterien für «gute» ${ }^{1}$ Psychotherapie. Da ethische Fragen und Konflikte in der Psychotherapie omnipräsent sind, ist eine Psychotherapie erst dann gut, wenn diese ethischen Aspekte einbezogen werden. Psychotherapie-Ethik als Wissenschaft vom moralischen Urteilen und Handeln in der Psychotherapie ist daher eine wichtige Voraussetzung für professionelles klinisches Arbeiten und sollte als Basiskompetenz von Psychotherapeuten systematisch vermittelt werden und.

\section{Ethik als notwendige Bedingung für gute Psychotherapie}

Die Diskussion der notwendigen Bedingungen einer guten Psychotherapie orientiert sich meistens primär an der Frage, welches therapeutische Vorgehen psychisches Leiden möglichst wirksam, möglichst schnell und möglichst kostengünstig reduzieren hilft. Diesbezüglich verläuft die fachliche und politische Debatte bisher vergleichbar zu anderen Bereichen der Medizin. Im Zentrum stehen die Kriterien der Wirksamkeit, Zweckmässigkeit und Wirtschaftlichkeit einer Behandlung wie sie im Deutschen Sozialgesetzbuch (SGB $\S 2$ und §12) als Voraussetzungen zur Kostenübernahme durch die Krankenversicherung genannt sind: «Qualität und Wirksamkeit der Leistungen haben dem allgemein anerkannten Stand der medizinischen Erkenntnisse zu entsprechen» und «Die Leistungen müssen ausreichend, zweckmäßig und wirtschaftlich sein“. In der Schweiz sind im Bundesgesetz über die Krankenversicherung (KVG) dieselben Voraussetzungen definiert: «Die Leistungen [...] müssen wirksam, zweckmässig und wirtschaftlich sein. Die Wirksamkeit muss nach wissenschaftlichen Methoden nachgewiesen sein.»

Im vorliegenden Artikel wird argumentiert, dass Wirksamkeit, Zweckmässigkeit und Wirtschaftlichkeit zwar notwendige, jedoch nicht hinreichende Kriterien für gute Psychotherapie sind. Gut ist eine Psychotherapie erst, wenn auch der Bereich der Ethik einbezogen wird. [1-2]

Tabelle: Die vier notwendigen Kriterien guter Psychotherapie

\begin{tabular}{l} 
Vier Kriterien guter Psychotherapie \\
\hline Wirksamkeit \\
\hline Zweckmässigkeit \\
\hline Wirtschaftlichkeit \\
\hline Ethik \\
\hline
\end{tabular}

\section{Ethische Herausforderungen sind in der Psychotherapie omnipräsent}

Betrachtet man das Spektrum psychotherapeutischer Situationen und Herausforderungen, so wird rasch klar, dass Reflexion und Berücksichtigung ethischer Fragen oft unumgänglich sind, um gute

\footnotetext{
${ }^{1}$ Zum Begriff des «Guten» im vorliegend gemeinten Sinne siehe Abschnitt zu Ethik und Moral.
} 
psychotherapeutische Entscheidungen fällen zu können. Einige Beispiele für Bereiche ethischer Herausforderungen in der Psychotherapie sind Art und Umfang der Patienteninformation, informierte Einwilligung in die Therapie, Einwilligungs- respektive Urteilsfähigkeit von Patientinnen und Patienten, Vertraulichkeit und Schweigepflicht, Interessenkonflikte des Therapeuten, körperlichen Berührungen, sexuelle Kontakte und andere Formen des Missbrauchs, Grenzen der therapeutischen Beziehung, Umgang mit eigenen Fehlern, kulturelle Differenzen und interkulturelle Kompetenzen, Online-Interventionen oder Klinikeinweisungen sowie Behandlungen gegen den Willen von Patientinnen und Patienten. [1-2; 3] Reflexion und Berücksichtigung solcher ethischer Fragen sind in der Psychotherapie nicht erst dann relevant, wenn moralisches Fehlverhalten bereits stattgefunden hat, sondern sie sind ,integraler Bestandteil der klinischen Praxis und Theorie, da Psychotherapie nicht nur die Behandlung von psychischen Symptomen beinhaltet, sondern die ganze Person in ihrem bio-psycho-sozialen Kontext“. [2, S. 21]

\section{Vier Fallvignetten}

Die vier Fallvignetten im folgenden Textkasten sollen zur Illustration dienen, dass ethische Herausforderungen in der Psychotherapie jederzeit und häufig unerwartet auftreten können, oft innert relativ kurzer Zeit eine Reflexion und anschliessende Entscheidung inklusive ethischer Begründung erfordern und nicht alleine auf der Basis klassischen psychotherapeutischen Wissens und Könnens befriedigend bewältigt werden können, sondern Kompetenzen in der Abwägung ethischer Argumente verlangen.

Die Fallvignetten stammen allesamt aus dem Buch «Ethics in Psychology and the Mental Health Professions. Standards and Cases» von Gerald P. Koocher (ehemaliger Präsident der American Psychological Association, APA) und Patricia Keith-Spiegel [4] und wurden für den vorliegenden Artikel moderat verändert ins Deutsche übersetzt.

\section{Textkasten}

Wo liegt die Grenze der Toleranz?

„Ich habe während der letzten vier Monate versucht objektiv und einfühlsam zu bleiben, aber es graut mir vor jeder Stunde mit Herrn Chauvin. Er äußert sich immer wieder rassistisch und sexistisch, was ich nicht tolerieren kann und weshalb ich ihn eigentlich nicht mehr behandeln möchte. Gleichzeitig sehe ich, dass er gravierende Probleme hat, die einer Psychotherapie bedürfen.“

\section{Wo liegt die Grenze der Schweigepflicht?}

Nachdem Frau Glock ihrem Ärger gegenüber ihrem Freund während der ganzen Psychotherapiesitzung Luft gemacht hat, funkeln ihre Augen vor Wut. Sie steht von ihrem Stuhl auf und flüstert bevor sie sich zur Tür dreht: „Er ist erledigt!“ Ich weiß, dass die Patientin zuhause eine Waffe hat.

Wo liegt die Grenze bei körperlichen Berührungen zwischen Patienten und Psychotherapeut? 
Frau Schnell, eine impulsive und oft übermütige Patientin, betritt meine Praxis, zieht ihr Smartphone aus der Tasche, schaltet Musik ein und ruft: „Ich habe gestern Abend einen Tango-Wettbewerb gewonnen!“ Sie hüpft zu mir, legt ihre Arme um mich und kreischt: „Lassen Sie uns tanzen!““

Wo liegt die Grenze einer professionellen Therapiebeziehung und ab wann besteht ein Interessenkonflikt?

„Mein Bruder Pius vermutet, dass seine 15-jährige Tochter Freya ungeschützten Sex mit verschiedenen Partnern hat und Drogen konsumiert. Er fragt mich, ob ich den Teenager psychotherapeutisch behandeln würde. Freya weigere sich, zu einem anderen Psychotherapeuten zu gehen; zu mir - ihrem Onkel - würde sie jedoch kommen. Pius ist zudem vermögend und würde mir ein hohes Honorar bezahlen. Ich könnte das Geld gut gebrauchen.“

\section{Ethik und Moral}

Seit Aristoteles wird Ethik als die philosophische Disziplin definiert, die sich mit den Kriterien für gutes Handeln und die Bewertung seiner Motive und Folgen befasst. [5] Alltagssprachlich ist der Begriff des Guten unscharf und kann Verschiedenes bedeuten, beispielsweise passend, geeignet, angemessen, hochwertig oder tauglich. Die Ethik befasst sich jedoch mit dem Guten aus philosophischer Sicht. Hierbei geht es nicht um das Zweckmässige, das Geeignete oder das Nützliche sondern um das Gute schlechthin. Das Gute in diesem Sinne ist das unbedingt Wünschenswerte und als richtig Befundene, nach dem sich unsere Handlungen richten sollen. Es ist diese Definition des Guten, die der vorliegenden Diskussion zugrunde liegt, was gute Psychotherapie ist.

Ethik beinhaltet die wissenschaftliche Auseinandersetzung mit moralischen Werthaltungen und gesellschaftlichen Normen. Der Begriff der Moral dagegen bezieht sich im Deutschen in der Regel auf individuelle Werthaltungen einer Person. Somit ist Ethik in diesem Sinne selbst nicht Moral; vielmehr ist Moral der Untersuchungsgegenstand der Ethik [1]. Medizinethik ist somit die Wissenschaft vom moralischen Urteilen und Handeln im Gesundheitswesen. [6] Entsprechend bezeichnet PsychotherapieEthik die Wissenschaft vom moralischen Urteilen und Handeln in der Psychotherapie.

\section{Deskriptive und normative Sätze}

Gemäss Humes Gesetz, dass es einen basalen Unterschied zwischen wertenden (normativen) und beschreibenden (deskriptiven) Sätzen gibt, wird in der Ethik normativ geklärt, wie etwas sein sollte (Was soll ich tun?), nicht wie etwas tatsächlich ist (Was tue ich?). Damit unterscheidet sich Ethik grundlegend von den empirischen Wissenschaften, das heisst, was gutes Handeln und die Bewertung seiner Motive und Folgen beinhaltet, kann prinzipiell nicht empirisch geklärt werden.

\section{Ethik als Basiskompetenz von Psychotherapeuten}


Das primäre Ziel der Förderung von Psychotherapie-Ethik ist nicht, dass Ethikexperten bei spezifischen ethischen Herausforderungen routinemäßig konsultiert werden, Vielmehr sollten moralische Sensibilität und Grundlagen in Psychotherapie-Ethik vermehrt zu Basiskompetenzen psychotherapeutischen Denkens und Handelns werden. Anders ausgedrückt heißt dies, dass Ethik nicht etwas ist, das von Psychotherapeuten standardmässig ausgelagert und delegiert werden sollte.

Bei besonders schwierigen moralischen Dilemmata sowie für Beratung und Supervision kann es jedoch hilfreich oder sogar unumgänglich sein, dass eine externe Fachperson für klinische Ethikberatung hinzugezogen wird, die in einer oder mehreren bestimmten Formen der Ethikkonsultation wie beispielsweise dem Modell der Moral Case Deliberation geschult ist. [7]

\section{Welche Ethikkompetenzen brauchen Psychotherapeuten}

Basiskompetenzen in Psychotherapie-Ethik beinhalten zwei Ebenen, erstens die Ebene des Wissens und zweitens die praktischen Ebene. Auf der Wissensebene ist eine Vertrautheit mit ethischen Grundbegriffen, unterschiedlichen Ethikkonzepten inhaltlicher und prozeduraler Art sowie relevanter Gesetze, berufsethischer Richtlinien und Kodizes nötig. [1] Wichtige Grundbegriffe umfassen beispielsweise die folgenden Begriffe: Ethik, Moral, Selbstbestimmung Gerechtigkeit, Würde und Güterabwägung. Bei den Ethikkonzepten sind normative Ethiktheorien wie der Utilitarismus als eine Form des Konsequentialismus, die Pflichtethik (Deontologie), die Prinzipienethik, die Tugendethik oder die Care-Ethik inklusive ihrer jeweiligen Vor- und Nachteile zentral.

Mindestens genauso wichtig wie ethisches Grundlagenwissen sind jedoch praktische Kompetenzen wie die Fähigkeit, moralische Fragen im Alltag zu identifizieren und zu artikulieren, was auch moralische Sensibilität genannt wird; [8] eigene moralische Positionen zu reflektieren, und argumentativ zu vertreten; die Sichtweisen und Interessen anderer Beteiligter - auch anderer Berufsgruppen im Gesundheitswesen zu erkennen und zu berücksichtigen; sowie Entscheidungen ethisch zu begründen, zu kommunizieren und umzusetzen. [9]

\section{Die Prinzipienethik nach Beauchamp und Childress und das reflexive Gleichgewicht}

Ein Ethikkonzept verdient vorliegend aufgrund seiner weltweiten Verbreitung in der Medizinethik besondere Aufmerksamkeit: die Prinzipienethik nach Tom Beauchamp und James Childress. [10] Deren zentrale Forderung ist, dass ethische Herausforderungen im Gesundheitswesen stets unter Bezugnahme auf die folgenden vier Prinzipien der biomedizinischen Ethik betrachtet werden sollten - Respekt vor der (Patienten-)Autonomie, Nichtschaden, Wohltun und Gerechtigkeit.

Bei der Beurteilung eines spezifischen ethischen Problems werden diese vier relativ abstrakten Prinzipien zuerst mit dem konkreten Inhalt des Problems angereichert und anschließend im Rahmen einer moralischen Güterabwägung gewichtet. 
Das Modell der Prinzipienethik ist als Orientierungsrahmen besonders attraktiv für die PsychotherapieEthik, «weil es für sich in Anspruch nimmt, grundsätzlich undogmatisch und offen gegenüber Verfechtern aller Ethiktheorien, liberal, transparent und rational zu sein». [1, S. 10; vgl. z.B. auch 11]

Auch die Idee des sogenannten reflexiven Gleichgewichts [12] soll vorliegend aufgrund seiner Bedeutung für die Psychotherapie-Ethik besonders hervorgehoben werden: «Wohlerwogene Einzelurteile können unser Verständnis von Prinzipien und übergeordneten Ethik-Theorien erhellen und umgekehrt, so dass ethische Reflexion sowohl ein induktives, fallbasiertes als auch ein deduktives, theoriegeleitetes Element umfasst». [1, S. 5]

\section{Nachholbedarf in der psychotherapeutischen Weiterbildung}

An den meisten Medizinischen Fakultäten gibt es mittlerweile Lehrstühle für Medizinethik und für Studierende der Humanmedizin sind obligatorische Ethikkurse inzwischen Standard. Parallel dazu wurden längst Lehrbücher für Medizinethik publiziert, die nicht nur auf grundlegende ethische Begriffe, Theorien und Argumente eingehen, sondern auch eine breite Palette an klinischen Fallbeispielen beinhalten sowie Modelle der ethischen Entscheidungsfindung vorstellen. [siehe z.B. 6]

Da ethische Fragen in der Psychotherapie omnipräsent sind, wurden ethische Themen in Fachkreisen schon immer diskutiert. Entsprechend befassen sich verschiedene Standesordnungen im Bereich der Psychotherapie mit ethischen Fragen und rufen Psychotherapeuten dazu auf, Verantwortung für eine sachgerechte Auseinandersetzung mit ethischen Fragen im Rahmen ihrer beruflichen Tätigkeit zu übernehmen. So fordert beispielsweise die European Federation of Psychologists' Associations (EFPA) im Meta Code of Ethics: [13] "Obligation to have a good knowledge of ethics, including the Ethical Code, and the integration of ethical issues with professional practice" (Art. 3.2.1) oder "Recognition that ethical dilemmas occur and responsibility is placed upon the psychologist to clarify such dilemmas and consult colleagues and/or the national Association, and inform relevant others of the demands of the Ethical Code" (Art. 3.3.6).

Konsistent mit der Wichtigkeit ethischer Aspekte in der Psychotherapie äußern Psychotherapeuten in Studien immer wieder einen erheblichen Bedarf an ethischer Orientierung in der Aus- und Weiterbildung sowie in der Supervision. [z.B. 14]

Im Kontrast dazu hat sich jedoch «die systematische Vermittlung von Psychotherapie-Ethik [in der Ausbildung von Psychotherapeuten] noch nicht durchgesetzt, obwohl der Bedarf klar gegeben wäre. Hier besteht ein klarer Nachholbedarf. Zudem existiert im deutschsprachigen Raum nur wenig geeignete Literatur als Basis- oder Nachschlagewerke für das Fach der Psychotherapie-Ethik». [1, S. 2]

\section{Fazit für die Praxis}

Psychotherapie-Ethik als Wissenschaft vom moralischen Urteilen und Handeln in der Psychotherapie ist eine wichtige Voraussetzung für professionelles klinisches Arbeiten und sollte als Basiskompetenz von Psychotherapeuten in jeder Psychotherapie-Weiterbildung systematisch sowohl auf der theoretischen 
(siehe z.B. das Lehrbuch «Psychotherapie-Ethik» von Trachsel, Gaab und Biller-Andorno [1]) als auch auf der praktischen Ebene mittels Übungen und gemeinsamer Diskussion ethischer Dilemmata in der Psychotherapie vermittelt werden.

\section{Literaturverzeichnis}

[1] Trachsel M, Gaab J, Biller-Andorno N. Psychotherapie-Ethik. Göttingen: Hogrefe; 2018

[2] Gaab J, Trachsel M. Was wirkt, ist nicht immer gut. Ethik als notwendiges Kriterium für eine gute Psychotherapie. Psychoscope 2019; 1: 19-21

[3] Trachsel M, grosse Holtforth M, Biller-Andorno N, Appelbaum, PS, Informed consent for psychotherapy: still not routine. The Lancet Psychiatry 2015; 2(9): 775-777.

[4] Koocher GP, Keith-Spiegel P. Ethics in psychology and the mental health professions. Standards and cases. Fourth Edition. Oxford: Oxford University Press; 2016

[5] Bien G, Hrsg. 1985. Aristoteles Nikomachische Ethik. Philosophische Bibliothek, Bd.5, Vierte Auflage. Hamburg: Felix Meiner Verlag; 1985

[6] Wiesemann C, Biller-Andorno N. Medizinethik. Stuttgart. Thieme; 2004

[7] Molewijk ACT, Abma M, Stolper M, Widdershoven G. Teaching ethics in the clinic. The theory and practice of moral case deliberation. Journal of Medical Ethics 2008; 34; 120-124.

[8] Reynolds SJ. Moral attentiveness: Who pays attention to the moral aspects of life? Journal of Applied Psychology 2008; 93(5): 1027-1041.

[9] Biller-Andorno N, Neitzke G, Frewer A, Wiesemann C. Lehrziele "Medizinethik im Medizinstudium". Ethik in der Medizin 2003; 15(2): 117-121.

[10] Beauchamp T, Childress J. Principles of biomedical ethics. Seventh Edition. Oxford: Oxford University Press; 2013

[11] Arras JD. The way we reason now: reflective equilibrium in bioethics. In: Steinbock B, Hrsg. The Oxford handbook of bioethics. Oxford: Oxford University Press; 2007: 46-71.

[12] Daniels N. Reflective equilibrium. In Zalta EN, Hrsg. The Stanford Encyclopedia of Philosophy; 2016. https://plato.stanford.edu/entries/reflective-equilibrium/ (zuletzt abgerufen am 25. April 2019).

[13] European Federation of Psychologists' Associations, EFPA. Meta Code of Ethics. Brüssel, Belgien: EFPA; 2015. http://www.efpa.eu/ethics/meta-code-of-ethics- (zuletzt abgerufen am 25. April 2019).

[14] Rabenschlag F, Steinauer R, Heimann, R, Reiter-Theil S. Pilotstudie: Wahrnehmung ethischer Aspekte in der psychiatrischen Patientenversorgung. Psychiatrische Praxis 2014; 41: 376-378.

\section{Kurzbiografie}


PD Dr. med. Dr. phil. Manuel Trachsel studierte Humanmedizin, Psychologie und Philosophie/Ethik an der Universität Bern. Er ist Privatdozent an der Medizinischen Fakultät der Universität Zürich, Oberassistent am Institut für Biomedizinische Ethik und Medizingeschichte der Universität Zürich und Oberarzt an den Psychiatrischen Diensten Thun. Manuel Trachsel unterrichtet Psychiatrie- und Psychotherapie-Ethik in verschiedenen Weiterbildungs-Studiengängen und ist Lead Editor des Oxford Handbook of Psychotherapy Ethics. Weitere Forschungsschwerpunkte bilden Ethik am Lebensende sowie Konzept und klinische Evaluation der Einwilligungsfähigkeit/Urteilsfähigkeit.

\section{CME-Fragen}

\section{Welches Prinzip gehört nicht zu den vier Prinzipien der biomedizinischen Ethik nach Beauchamp} und Childress?
a) Gerechtigkeit
b) Respekt vor der Autonomie
c) Nichtschaden
d) Würde
e) Wohltun

\section{Welcher der folgenden ist ein normativer Satz?}

a) Psychotherapie benötigt eine teure Infrastruktur.

b) Nur empirisch validierte Psychotherapien zuzulassen, ist auch aus ökonomischer Sicht sinnvoll.

c) Je seltener bestimmte Patientengruppen behandelt werden, desto höher ist das Risiko, als Therapeut einen Fehler zu begehen.

d) Durch die Konzentration an weniger Standorten lässt sich eine höhere Qualität und Wirtschaftlichkeit von Psychotherapie erreichen.

e) Psychotherapie sollte in Deutschland nur an Universitätskliniken angeboten werden.

\section{Welche Aussage ist falsch?}

a) Ethik beinhaltet die wissenschaftliche Auseinandersetzung mit moralischen Werthaltungen und gesellschaftlichen Normen.

b) Moral Case Deliberation ist eine Form der klinischen Ethikberatung.

c) Moralische Sensibilität bezeichnet die Fähigkeit, moralische Fragen im Alltag zu identifizieren und zu beantworten.

d) Wirksamkeit, Zweckmässigkeit und Wirtschaftlichkeit einer Behandlung sind Voraussetzungen, damit diese von der Krankenversicherung vergütet wird.

e) Wohlerwogene Einzelurteile können unser Verständnis von Prinzipien und übergeordneten Ethik-Theorien erhellen und umgekehrt, so dass ethische Reflexion sowohl ein induktives, fallbasiertes als auch ein deduktives, theoriegeleitetes Element umfasst. 